M. A. Smith, H. F. Henrichs, and J. Fabregat, eds.

\title{
On the Excitation Mechanism for the Nonradial Pulsations in $\epsilon$ Persei
}

\author{
Eiji Kambe \\ Dept. of Geoscience, Nat'l Defence Acad., Yokosuka, Kanagawa \\ 239-8686, Japan
}

\section{Hideyuki Saio \& Umin Lee}

Astron. Inst., School of Sci., Tohoku Univ. Sendai 980-8578, Japan

\begin{abstract}
We have examined whether the kappa-mechanism at the " $Z$ " opacity bump can excite overstable nonradial pulsation (NRP) modes such as those responsible for the line-profile variations $(l p v)$ observed for $\epsilon$ Persei (Gies et al. 1999).

From the $l p v$ modeling with realistic stellar parameters and the stateof-the-art codes, we confirmed that the observed $l p v$ can generally be explained by the modes with $l$ values suggested by Gies et al. (1999). We have also found that some tesseral modes, which have smaller $m$ than those of sectoral modes, can reproduce the observed $l p v$ well for the periods of $3.51 \mathrm{hr} / 3.84 \mathrm{hr}$ and $4.06 \mathrm{hr} / 4.54 \mathrm{hr}$. Our linear non-adiabatic nonradial pulsation analysis applied to massive $\left(13 \mathrm{M}_{\odot}--14 \mathrm{M}_{\odot}\right)$ stars shows that the periods of higher $|m|$ values in the co-rotating frame are too long to be excited by the Z-bump kappa-mechanism while the smaller $|m|$ values give marginal consistency.
\end{abstract}

\section{Introduction}

$\epsilon$ Persei (HD 24760, ADS 2888A) is an early-type (B0.5 - 0.7 IV - III) variable star. It shows remarkable line-profile variations $(l p v)$, in which the features (or bumps) travel from blue to red (e.g., Smith Fullerton, \& Percy 1987, Gies \& Kullavanijaya 1988, Gies et al. 1999). In addition to the $l p v$, it shows photometric variations with similar periods (Smith et al. 1987).

During the week-long campaign in 1996 January, Gies et al. (1999) monitored the UV (by IUE), He I $\lambda 6678$, and $\mathrm{H} \alpha$ lines. From the UV data, which only have a 3 hour gap in the time sampling each day, they found at least six periods in the $l p v$. In Figure 1, we plotted each frequency component of the $l p v$ for the main two periods of $3.84 \mathrm{hr}$ and $4.06 \mathrm{hr}$. The UV data are given only as the UV cross correlation function (ccf) values in Gies et al. (1999) and the gray scale in the figure corresponds to the one when the central line depth is about 0.423 of the continuum. In $\epsilon$ Per, the observed pattern for each frequency component travels continuously from blue to red for all peaks. Such features are also seen in $\zeta$ Oph whereas more complicated $l p v$ are observed in some stars like $\lambda$ Eri. 

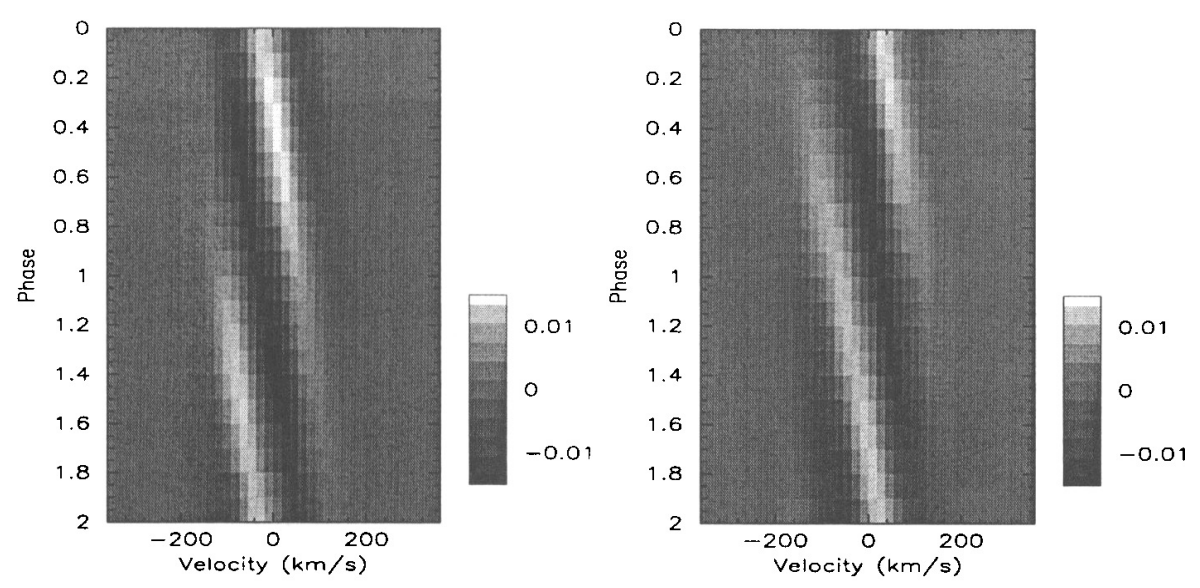

Figure 1. The $l p v$ for each frequency component by Gies et al. (1999) November; (a) for the $3.84 \mathrm{hr}$ period; (b) for the $4.06 \mathrm{hr}$ period.

In this paper we discuss mainly the overstability of the shorter five periods ranging from 2.3 to $4.5 \mathrm{hr}$. The extensive analysis of the modal nature of $\epsilon$ Per will be presented in a separate paper.

\section{The $1 p v$ Calculations}

\subsection{Basic Stellar Parameters and the Codes}

We use $T\left(=T_{\text {pol }}\right)=27,600 \mathrm{~K}, \log g\left(=\log g_{\mathrm{eq}}\right)=3.85, R\left(=R_{\mathrm{eq}}\right)=6.9 \mathrm{R}_{\odot}$ (Tarasov et al. 1995), $\Omega_{\text {rot }}=0.6 \mathrm{~d}^{-1}$ (Gies et al. 1999) and $V_{\mathrm{e}} \sin i=134 \mathrm{~km} \mathrm{~s}^{-1}$. Assuming Roche model, these values give $M=13.7 \mathrm{M}_{\odot}$. It is close to the one by Tarasov $\left(M=13.5 \mathrm{M}_{\odot}\right)$. The luminosity, $\log L / L_{\odot}=4.31$, is slightly lower than that by Tarasov $\left(\log L / L_{\odot}=4.40\right)$ but is consistent with that derived from HIPPARCOS data $\left(\log L / L_{\odot}=4.26\right)$.

We use the BRUCE code (Townsend 1997) to calculate lpv's caused by spheroidal modes (see Kambe et al. 1999 in detail). Since the intrinsic profile and the temperature dependence of the UV cross correlation functions are not clearly known, we use the He I $\lambda 6678$ line for the $l p v$ modeling. A Kurucz model atmosphere (1994 CD-ROMs) is used to estimate the flux variation at $500 \mathrm{~nm}$.

\subsection{Results}

In Table 1 we list the detected five periods as well as the $l$ values suggested by Gies et al. (1999). Assuming $\Omega_{\text {rot }}=0.6 \mathrm{~d}^{-1}$ and assigning $m$ values differing by one (to be consistent with the $\Omega_{\text {rot }}$ ) between modes (2) and (3) and between (4) and (5), we found that the observed features for the five periods can well be explained by the modal $l$ values given by Gies et al. For the modes with $3.51 \mathrm{hr} / 3.84 \mathrm{hr}$ and $4.06 \mathrm{hr} / 4.54 \mathrm{hr}$ periods, the $|m|=l-1 /|m|=l-2$ modes as well as $|m|=l /|m|=l-1$ modes can reproduce the observed $l p v$ well. For such high frequency modes, the radial velocity perturbations dominate the $l p v$. The 


\begin{tabular}{c|ccc|ccc|ccc} 
Table 1. & \multicolumn{1}{c}{ Observed periods and periods expected on the star } \\
\hline & \multicolumn{3}{|c}{ Gies et al } & \multicolumn{3}{c}{ case A } & \multicolumn{5}{c}{ case B } \\
& $\begin{array}{c}\text { Freq. } \\
\left(\mathrm{d}^{-1}\right)\end{array}$ & $\begin{array}{c}\text { Period } \\
(\mathrm{hr})\end{array}$ & $l$ & $m$ & $\begin{array}{c}\text { Freq. } \\
\left(\mathrm{d}^{-1}\right)\end{array}$ & $\begin{array}{c}\text { Period } \\
(\mathrm{hr})\end{array}$ & $m$ & $\begin{array}{c}\text { Freq. } \\
\left(\mathrm{d}^{-1}\right)\end{array}$ & $\begin{array}{c}\text { Period } \\
(\mathrm{hr})\end{array}$ \\
\hline$(1)$ & 10.58 & 2.26 & 5 & -5 & 7.58 & 3.17 & & & \\
$(2)$ & 6.84 & 3.51 & 4 & -4 & 4.44 & 5.41 & -3 & 5.04 & 4.76 \\
$(3)$ & 6.25 & 3.84 & 4 & -3 & 4.45 & 5.39 & -2 & 5.05 & 4.75 \\
$(4)$ & 5.91 & 4.06 & 3 & -3 & 4.11 & 5.84 & -2 & 4.71 & 5.10 \\
$(5)$ & 5.28 & 4.54 & 3 & -2 & 4.08 & 5.88 & -1 & 4.68 & 5.13 \\
\hline
\end{tabular}

radial velocity amplitudes of the $\mathrm{P}=4.06 \mathrm{hr}$ mode can be estimated from the amplitudes of $\operatorname{He} \lambda 6678$ line and it is $28 \mathrm{~km} \mathrm{~s}^{-1}$ for the $l=3, m=-3$ mode and $22 \mathrm{~km} \mathrm{~s}^{-1}$ for the $l=3, m=-2$ mode. The expected flux variations at $500 \mathrm{~nm}$ are $7 \mathrm{mmag}$ for the $l=3, m=-3$ mode and $21 \mathrm{mmag}$ for the $l=3, m=-2$ mode, which is consistent with previous observations (Smith et al. 1987). The simultaneous observations both with photometry and spectroscopy might be useful for the further mode identification.

\section{NRP Stability Analysis}

\subsection{Evolutionary Models}

We have calculated evolutionary models of $13 M_{\odot} \sim 14 M_{\odot}$ from chemically homogeneous models to central hydrogen exhaustion phases. A Henyey-type evolution code has been employed with OPAL95 tables (Iglesias \& Rogers 1996) and nuclear reaction rates from Caughlan \& Fowler (1988). We have treated the convective and semi-convective mixing by solving diffusion equations adopting the diffusion coefficient prescribed by Spruit (1992). We have neglected the effect of rotation. We have adopted hydrogen abundances slightly smaller than the standard value 0.7 , because recent abundance analysis by Tarasov et al.(1995) indicates a high helium abundance of $\mathrm{He} / \mathrm{H}=0.23$.

\subsection{Unstable Modes}

Figure 2 shows periods of overstable modes for $l=0$ to $l=6$ for the $M=14 \mathrm{M}_{\odot}$, $\mathrm{X}=0.65$, and $\mathrm{Z}=0.02$ model. The blue edge of instability boundary tends to be slightly bluer for higher $l$ modes. As the effective temperature decreases, the longest period and the number of overstable modes increase.

For the periods (2) - (5), higher $|m|$ values give longer periods in the frame co-rotating with the stellar surface (Table 1), which happened to be too long to be excited by the Z-bump kappa-mechanism. Although the number of overstable modes, the blue edge of the instability region, and the shortest period of overstable modes at a given $T_{\text {eff }}$ is affected by $Z$ value, the longest period is insensitive to $Z$. This property can be attributed to the fact that the radiative dissipation in the core is significant in these modes because the amplitude of a low-frequency mode rapidly oscillates spatially in the core (Gautschy \& Saio 1993). The dissipation effect for a given period is insensitive to $Z$ because $N^{2}$ is mainly determined by the gradient of hydrogen abundance. For a given mass, the longest unstable period becomes longer for a higher helium abundance, 


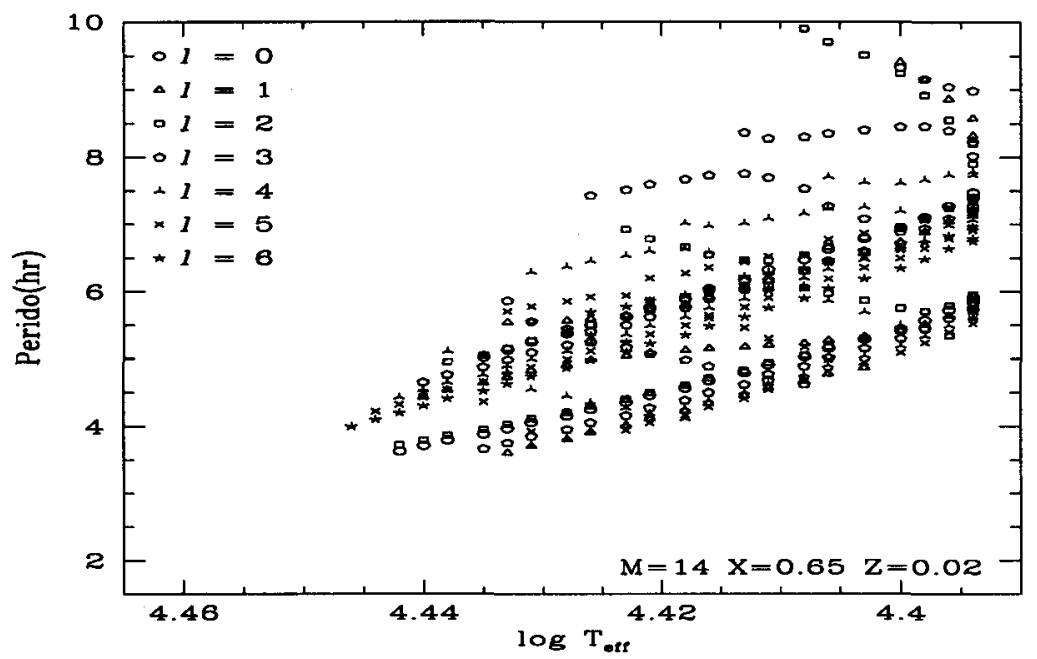

Figure 2. Periods of excited modes versus effective temperature for the model $\left(\mathrm{M}=14 \mathrm{M}_{\odot}, \mathrm{X}=0.65, \mathrm{Z}=0.02\right)$

since the thermal time scale of the core then becomes longer. In a such model, however, we must decrease the mass to prevent the effective temperatuere and luminosity from being too high. The end result of these adjustments is that the longest unstable period is affected only little by the initial helium abundance.

Those modes with smaller $|m|$ values give marginal consistency to our stability analysis. Nonetheless, some important questions arise from this work on $\epsilon$ Per that require attention: why are only marginally unstable modes excited?, and why are only retrograde modes stabilized.

\section{References}

Caughlan G.R., \& Fowler W.A. 1988, Atomic Data Nuclear Data Tables 40, 283 Gautschy A., \& Saio H. 1993, MNRAS 262, 213

Gies D.R., \& Kullavanijaya A. 1988, ApJ 326, 813

Gies D.R. et al 1999, ApJ, in press

Iglesias C.A., \& Rogers F.J. 1996, ApJ 464, 943

Kambe et al. 1999, this proceedings

Smith M.A., Fullerton A.W., \& Percy J.R. 1987, ApJ 320, 768

Spruit H.C. 1992, A\&A 253, 131

Tarasov A.E. et al. 1995, A\&AS 110, 59

Townsend, R.H.D. 1997, Ph D thesis, Univ. College London 